\title{
Personal Protective Equipment to an "Emotional Protective Environment": Reflection of a Pediatric Dentist amidst the Pandemic!
}

\author{
Ghousia Begum S
}

\begin{abstract}
Aim and objective: Emphasis on reflective practice in pediatric dentistry on implementing "Emotional Protective Environment" (EPE) while donning Personal Protective Equipment (PPE).

Background: The concept of reflective practice is seldom talked about in dentistry and is still in its budding stages. Reflecting on every case in my routine clinical practice turned my personal experiences into invaluable precious learnings; helped me to understand my strengths, weaknesses; questioned my underlying values, identified my personal limitations or areas for improvement.

Conclusion: Reflection does demand from clinicians, a dare to accept individual's limitations and uneasiness encountered during clinical practice by unmasking it all while simultaneously inviting others to view the prudence when you yourself are looking into the mirror of self-image. Reflective practice takes time from the hindsight experiences of the clinical practices in formulating and executing fruitful insights.

Clinical significance: We must work on Self-distancing (Adaptive self-reflection) while implementing the "Social Distancing" in professions dealing with children. Time for some earnest reasoning on visualizing our thoughts on some kind of reflector glass of minds in promoting the delivery of an Emotional Protective Environment along with the practice of Personal Protective Equipment!

Keywords: Emotional protective environment, Emotional contagion, Personal play therapy equipment, Pretend play dentist toys, Reflective practice, Tell show wear.

CODS Journal of Dentistry (2020): 10.5005/jp-journals-10063-0059
\end{abstract}

\section{Reflective Practice in Dentistry}

The concept of reflective practice is seldom talked about in dentistry and is still in its budding stages. Reflecting on every case in my routine clinical practice turned my personal experiences into invaluable precious learnings; helped me to understand my strengths, weaknesses; questioned my underlying values, identified my personal limitations or areas for improvement. Reflection on cases while pursuing Palliative care Fellowship [Kidwai Oncology Institute, Bengaluru], soon became habitual to me even at my Dental Care Unit. Reflection helps us to re-appreciate the job we do. ${ }^{1}$ While reflective practice being a widely discussed chapter in medical; I as a pediatric dentist inclined towards inclusive special needs dentistry could not emphasize enough, how important its inculcation in the dental curriculum would do.

Based on the latest series of events of the American Dental Association (ADA), which seem to respectfully and yet strongly disagree with the World Health Organization's statements whether the linking of COVID-19 with dentistry is evidence or non-evidence-based; the ADA by openly and quite assertively declared Dentistry as an Essential Health Care as on August 12, $2020{ }^{2}$ paving way for every dental practitioner to accommodate themselves while embracing a whole "new routine" which is a stark departure from the previous historic styles of routine dental practice. In hindsight, the last such pandemic and epidemic association of dentistry with HIV/AIDS was closely knit like the current on-going pandemic era. Progressing toward better healthcare, ADA's concluding statement just came onto rescuing stating - "dental care to be continued with appropriate personal
Department of Pedodontics and Preventive Dentistry (Inclusive Special Needs Dentistry), Dental Health Care and Research Unit @Gharonda [HOSMAT], Bengaluru, Karnataka, India

Corresponding Author: Ghousia Begum S, Department of Pedodontics and Preventive Dentistry (Inclusive Special Needs Dentistry), Dental Health Care and Research Unit @Gharonda [HOSMAT], Bengaluru, Karnataka, India, Phone: +91 9880775708, e-mail: drghousia786@ gmail.com

How to cite this article: Begum SG. Personal Protective Equipment to an "Emotional Protective Environment": Reflection of a Pediatric Dentist amidst the Pandemic!. CODS J Dent 2020;12(1):21-23.

Source of support: Nil

Conflict of interest: None

protective equipment (PPE) irrespective of global pandemics or other disaster situations".

Amidst the chaos, entailing reflection, I step aside to re-visit this extraordinary encounter with a pandemic as a perfect chance that could have ever prepared me nothing more earnestly than any of the academic qualifications. All and anything positive along with negative should be reflected upon, making reflection a normal practice among dental professionals helping them to foster improvements in their practice. A joint statement by the General Dental Council of UK and eight other healthcare organizations on the importance and benefits of being a Reflective practitioner were regulated and published on June 18, 2019. ${ }^{3}$ It also assures the patients that the dental team is in the progressive process of evolving by analyzing events that went well as well the ones that did not go as planned. 


\section{The Much Talked About Personal Protective Equipment}

Like any fancy-schmancy phrase, "Personal Protective Equipment, or PPE", its Donning-Doffing since pandemic has become part of our day-to-day lexicon expanding its use beyond the healthcare professionals to regular people. The origin of PPE has its way back during the war years as a means to prevent contamination from chemical warfare. Leonardo da Vinci is the original inventor of the respirator during the 16th century after which the respiratory protection technology has been standardized and requirements clearly spelled out safety device by the National Institute for Occupational and Safety (NIOSH) and the Occupational Safety and Health Agency (OSHA). ${ }^{4}$

Personal protective equipment has evolved over the centuries. Dental care is one of the "uncontrolled environments" with challenging or unpredictable behavioral patients at times. It is imperative to utilize the PPE effectively for maximum infection prevention. This presents the dental fraternity as more dignified health care providers of the society while enhancing their professionalism via more advanced and sophisticated elements. All of it including efforts of upgradations gives cues to patients about high-quality dental care for themselves.

\section{The Challenging Part-Adaptive Behavioral Support Etiquettes}

Personal protective equipment renders one physically safe but poses challenges for emotional safety at the same time. It is not just me as a pediatric dentist going through this paranoia; occupational therapists (oro-motor in particular), speech-language pathologists too are going through this feeling of fear of being separated from their child clientele as they assess swallowing and voice difficulties. Social distancing just does not blend well with any profession that involves children. The PPE therefore should help in insulating rather than isolating.

The impact of communication skills cannot be underplayed that exist between dentists and their inexperienced patients or the children in easing their anxiety. Emotional distance may not be justifiable in care services. The emotional intelligence quotient involved in treating patients is an absolute obvious nuance in communication skills as identified by clinicians. The revolutionary change in the appearance of dentists can have an anxiety-inducing situation. Donning of PPE can therefore be undertaken after a warm greeting and hearty interviewing of the patient as suggested by Glaros and Gadbury-Amyot. ${ }^{5}$

\section{Emotional Protective Environment-The EPE!}

"Emotional contagion" refers to a phenomenon of an automatic adoption of the emotional state of another person. It is the tendency to automatically mimic and synchronize the facial expressions, vocalization postures, and movements with those of another person and, consequently, to converge emotionally as mentioned. ${ }^{6}$ It is particularly prevalent in health care settings, partly from patients but especially from staff. Emotions go "viral" causing a rippling effect. It is a proven fact that the merriment of one person has its influence on people up to three degrees away. ${ }^{7}$

Emotional broadcasting theory (EBT) proposes that the intrapsychic need to share experiences with others serves the interpersonal function of transmitting news. The power of positivity far outweighs the power of negativity. Hence, being on guard as to what emotions to broadcast as a dental care practitioner should be carefully weighed as it shall travel past the whole dental team as well as the patients being treated in the operatory. ${ }^{8}$

\section{Personal Play Therapy Equipment-A Different Personal Protective Equipment}

Introducing "Pretend Play-Pretend Dentist Toys" in the waiting area to connect to the child emotionally is a very helpful approach in connecting to the child emotionally and ensuring the healing they deserve from dental ailments. Allow each child their own "PPEpersonal play therapy equipment", to avoid the risk of infection, each child will have their very own play therapy box waiting on them on their appointment day at the dental operatory to be used only by them and their families to desensitize the whole new environment of infection protocol and allows a pro-social behavior in the operatory.

\section{"Tell-Show-Wear"Technique: Latent Inhibition Hypothesis}

A series of painless appointments till an invasive event renders less anxiety can be implemented by modes of Modeling visually in pamphlets, video displays in the waiting area to transmute unhelpful behaviors of children in a non-judgmental and un-threatening way. Fear, they say has the biggest eyes of all and can be easily combated by simply showing each piece of PPE along with an explanation with the "Tell-show-wear" technique before Donning PPE. This technique familiarizes and desensitizes anticipatory anxiety by diminishing and alleviating the fear of the unknown.

\section{Non-verbal: Sign Language}

The physical attire of the dentist should not project a mind-body separation. Transparent masks (FDA approved masks, such as, Safe " $\mathrm{N}$ " Clear) for lip reading, slowing hand movements down while signaling and using other facial grammatical markers to convey the messages with eyebrows. New Etiquettes in the welcoming of the child by the dental team staff by incorporating Touchless greetings like Air hugs, Elbow bumps, contactless high-fives, foot-based "Wuhan shakes" can create a fun and humorous atmosphere.

Donning and Doffing of emotions needs to be carefully practiced as we welcome the child patients with amplified enthusiasm, extrasparkle in the eyes and likewise managing goodbyes by the end of it all as we provide an emotional buffer as we allow them to part with some wow-some experiences as they leave the dental operatory. ${ }^{9}$

\section{Credo for Ethical Reflective Dental Practice}

"Reflective practice is not without moral significance ${ }^{\text {". }}{ }^{10}$ It does at times become an excruciatingly taxing affaire emotionally while judging ourselves during negative experiences or overwhelming while re-experiencing our positive outcomes. Doubtlessly it is a public display of emotions as practitioner's vulnerabilities, helplessness's, guilt-shame along with few overwhelming accolades as well. It is always important to keep guard that amidst self-realization of feelings, exists a third party involved as in patient privacy, consent, and safekeeping confidants. ${ }^{11}$ As a reflective practitioner it is important to respect the dignity of our nonconsenting patients by safeguarding their dignities as well while we self-talk to share our guilt, shame, or accolades with ourselves as well as our fellow colleagues. A fine-tuning of balanced fairness 
of ethics of care as sentimental workers had to be maintained to make it a wholesome soulful experience..$^{11,12}$

Though it seems to be a "confessional mode", it is for selfimprovement for better health care practices.

The aim of improvement through reflection was bequeathed to education from the Enlightenment 'Sapere aude! "Have courage to use your own reason!"-i.e., the motto of enlightenment', as aptly quoted by Immanuel Kant in 1785. ${ }^{12,13}$

\section{Concluding and After-thoughts}

Although this pandemic did bring hardships along its way, it has also inspired generousness and accommodative tolerance. A day is not too far to anticipate that PPE becoming an integral part of dentistry, eventually leading us to patients who would visualize the dentist with no protective garb as a warning to the welfare and maybe one of the reasons to invoke anxiety!

As I come to the inference of my overview, I am reminded of the popular John Dewey's quote-“We do NOT learn by Experience but, we learn from Reflecting on Experience".

Reflection does demand from clinicians, a dare to accept individual's limitations and uneasiness encountered during clinical practice by unmasking it all while simultaneously inviting others to view the prudence when you yourself are looking into the mirror of self-image. Reflective practice takes time from the hindsight experiences of the clinical practices in formulating and executing fruitful insights. Despite it being considered by many in the fraternity as a "non-clinical skill", I personally consider it crucial in the self-growth individually and professionally in honing clinical reasoning skills from pre-reflection to reflection stages. We must work on Self-distancing (Adaptive self-reflection) while implementing the "Social Distancing" in professions dealing with children. Undoubtedly, the greatest strength of any practitioner working with children lies in creating a compassionate link but at the same time rendering us the most vulnerable. Time for some earnest reasoning on visualizing our thoughts on some kind of reflector glass of minds in promoting the delivery of an Emotional Protective Environment along with the practice of Personal Protective Equipment!

\section{References}

1. Glaze J. Reflection as a transforming process: students advanced nurse practitioners' experiences of developing reflective skills as part of an MSc programme. J Adv Nurs 2001;34(5):639-647. DOI: 10.1046/j.1365-2648.2001.01793.x.

2. Available at: http://www.ada.org.>news-releases $>2020$-archives $>$ august.

3. Available at: http://www.gdc-uk.org>education-cpd.

4. Available at: http://www.cdc.gov >niosh.

5. Glaros AG, Gadbury-Amyot CC. How personal protective equipment affects perceptions of dentists. J Am Dent Assoc 1993;124(10):82-90. DOI: 10.14219/jada.archive.1993.0214.

6. Hatfield E, Cacioppo JT, Rapson RL. Studies in emotion and social interaction. Emotional contagion. Cambridge University Press, Editions de la Maison des Sciences 'Homme 1994.

7. Petitta L, Jiang L, Hartel CEJ. Emotional contagion and burnout among nurses and doctors: do joy and anger from different sources of stake holders matter? Stress \& Health 2017;33(4):358-369. DOI: 10.1002/ smi.2724.

8. Harber KD, Cohen DJ. The emotional brodcaster theory of social sharing. J Lang Soc Psychol 2005;24(4):382-400. DOI: 10.1177/0261927X05281426.

9. Isabella G, Carvalho. HC. Emotional contagion and socialization. Emot Technol Behav 2016.

10. Hargreaves J. Using patients: exploring the ethical dimension of reflective practice in nurse education. J Adv Nursing 1997;25(2):223228. DOI: $10.1046 /$ j.1365-2648.1997.1997025223.x.

11. Ghaye T. Is reflective practice ethical? (The case of the reflective portfolio). Reflect Pract: Int Multidisciplin Perspect 2007;8(2):151-162 DOI: 10.1080/14623940701288859.

12. Pollard V. Ethics and reflective practice: continuing the conversation. Reflect Pract: Int Multidisciplin Perspect 2008;9(4):399-407. DOI: 10.1080/14623940802431788.

13. Available at: www.columbia.edu. 